\title{
Volume guarantee ventilation in neonates treated with hypothermia for hypoxic-ischemic encephalopathy during interhospital transport
}

\author{
Lajos Lantos $^{1,2} \cdot$ Aniko Berenyi $^{1} \cdot$ Colin $_{\text {Morley }}^{2} \cdot$ Zsolt Somogyvari $^{1} \cdot$ Gusztav Belteki $\left({ }^{1,2}\right.$
}

Received: 20 March 2020 / Revised: 24 August 2020 / Accepted: 14 September 2020 / Published online: 25 September 2020

(c) The Author(s), under exclusive licence to Springer Nature America, Inc. 2020

\begin{abstract}
Objective We investigated if volume guarantee (VG) ventilation in babies with hypoxic-ischemic encephalopathy (HIE) during interhospital transport decreases tidal volumes and prevents hypocapnia.

Study design We computationally collected and analyzed ventilator data of babies ventilated with synchronized intermittent mandatory ventilation (SIMV) with VG $(n=28)$ or without VG $(n=8)$.

Result The expiratory tidal volume of ventilator inflations was lower with SIMV-VG (median [IQR]: 4.9 [4.6-5.3] mL/kg) than with SIMV only (median [IQR]: $7.1[5.3-8.0] \mathrm{mL} / \mathrm{kg}, p=0.01$ ). Babies receiving SIMV-VG had lower peak inflating pressures (median: $10.7 \mathrm{cmH}_{2} \mathrm{O}$, versus $17.5 \mathrm{cmH}_{2} \mathrm{O}, p=0.01$ ). There was no significant difference in minute ventilation or in $\mathrm{pCO}_{2}$. Babies with strong spontaneous breathing had a mean $\mathrm{PIP}<10 \mathrm{cmH}_{2} \mathrm{O}$ but this did not result in adverse events or worsening of acidosis.

Conclusions The use of VG ventilation in babies with HIE reduces tidal volumes and frequently results in very low inflating pressures without affecting $\mathrm{pCO}_{2}$.
\end{abstract}

\section{Introduction}

Hypoxic-ischemic encephalopathy (HIE) of the newborn is brain injury due to lack of oxygen around birth [1]. Despite many advances in obstetric and neonatal intensive care it remains a cause of significant mortality and long-term morbidity [2,3]. Therapeutic hypothermia for term and late preterm infants with moderate to severe HIE reduces mortality and neurodevelopmental disability [4] and the protective effect is maintained throughout childhood [5].

Beside therapeutic hypothermia, optimal intensive care support is essential to improve neurodevelopmental outcome and to prevent injury progression. Respiratory

Supplementary information The online version of this article (https:// doi.org/10.1038/s41372-020-00823-8) contains supplementary material, which is available to authorized users.

Gusztav Belteki

gbelteki@aol.com

1 Neonatal Emergency and Transport Service of the Peter Cerny Foundation, Budapest, Hungary

2 Neonatal Intensive Care Unit, The Rosie Hospital, Cambridge University Hospitals NHS Foundation Trust, Cambridge, UK management of these babies poses a challenge [6]. The majority of them receive mechanical ventilation [7] for apnea or seizures, respiratory or cardiovascular comorbidities or sedative effects of anticonvulsant medications. There is a significant risk of hypocapnia due to lower $\mathrm{CO}_{2}$ production caused by the lower metabolic rate and to hyperventilation caused by metabolic acidosis [8]. Hypocapnia is associated with poor neurodevelopmental outcome $[9,10]$. While a causative relationship has not been fully proven [6], several mechanisms have been suggested including cerebral vasoconstriction, reduced cerebral blood flow, increased neuronal excitability facilitating apoptotic cell death and seizures [11-13]. Maintaining normocapnia is important because hypercapnia may also be deleterious [14].

Volume targeted ventilation, also known as volume guarantee (VG), has been used increasingly in neonatal intensive care [15]. Systematic reviews comparing VG with standard pressure limited ventilation have shown that VG ventilation has tighter tidal volume and carbon dioxide control, lower mortality or bronchopulmonary dysplasia, fewer pneumothoraces and serious intraventricular hemorrhage, and fewer days of ventilation [16, 17]. However, most studies recruited very preterm infants and the benefits of VG ventilation in term babies are much less well 
established. In a randomized study of 40 infants born at $>34$ weeks of gestation Bhat et al. found fewer episodes of hypocapnia during VG but no other benefits; however, the infants did not have HIE [18].

Maintaining tidal volume during VG in hyperventilating infants with Kussmaul breathing can be difficult. If the baby is taking a strong breath during a ventilator inflation and the inspiratory tidal volume significantly exceeds the target VT, many VG ventilators immediately reduce the pressure to PEEP level and end ventilator inflation. However, the baby can continue breathing from the continuous gas flow in the ventilator circuit. Moreover, VG ventilation in babies with normal lungs and breathing above the set tidal volume causes the PIP to be reduced to just above the PEEP level, making the ventilator's driving pressure (PIP-PEEP), and hence its contribution to the work of breathing, minimal [19]. It has been suggested that this may cause exhaustion and acidosis [20] although we found no report presenting original data to support this hypothesis.

We were interested in the tidal volumes and inflating pressures during VG ventilation of babies undergoing therapeutic hypothermia for HIE and how these parameters correlated with the spontaneous breathing effort of these infants. We hypothesized that despite the above considerations the use of VG will reduce tidal volumes and the incidence of hypocapnia. We also reasoned that the use of VG in these babies will result in low PIPs used by the ventilator, but their use will not have deleterious effects.

\section{Subjects and methods}

\section{Patients}

Clinical and ventilator data were collected from all infants transferred by the Neonatal Emergency and Transport Service of the Peter Cerny Foundation (NETS-PCA, Budapest, Hungary) over a 29-month period (between 20 March 2017 and 4 September 2019) who received invasive or noninvasive respiratory support during interhospital transport using a fabian + ncpap evolution neonatal ventilator (Acutronic Medical Systems, Hirzel, Switzerland, software version: 4.0.1). The transport team comprised a fully trained neonatologist experienced in neonatal transport and an experienced neonatal transport nurse practitioner. Respiratory management, including the choice of ventilator mode and settings, was at the discretion of the transport team without an explicit protocol.

Of the 667 infants transferred during the period, we enrolled babies who were born after 36 completed weeks of gestation, received therapeutic hypothermia for HIE and were ventilated via an endotracheal tube during the transfer which lasted for at least $15 \min (n=49)$. Eligibility criteria for introducing therapeutic hypothermia was in accordance with the total body hypothermia for neonatal encephalopathy trial protocol [21]. Prior to transfer all infants received passive hypothermia at the referring unit which included reducing or turning off the heat source of the incubator or the resuscitation equipment and monitoring the rectal temperature. We excluded two infants who had major congenital abnormalities and one atypical case where hypothermia and transfer happened after a postnatal collapse at $20 \mathrm{~h}$ of age. Of the remaining 46 infants, we analyzed clinical and ventilator data from those receiving synchronized intermittent mandatory ventilation (SIMV) with VG $(n=28)$ or without VG $(n=$ $8)$. The transport service's guideline suggests the use of SIMV in infants suffering from HIE; the choice of whether to use VG or not was left to the clinician during the study period. When VG was used, the guideline was to use $4-6 \mathrm{~mL} / \mathrm{kg}$ as target tidal volume, but clinicians could reduce that below $4 \mathrm{~mL} / \mathrm{kg}$ if needed to avoid or reduce hypocapnia. The ten infants ventilated with other modes (SIPPV with or without VG or SIMV-VG with pressure support) were excluded. The study was approved by the Scientific and Medical Research Council Ethics Committee of Hungary (reference number: 40158/2018/EKU). All procedures were performed in accordance with the ethical standards of the Research Ethics Committee and the amended Helsinki Declaration (1983).

\section{Data retrieval}

We downloaded ventilator data using a data logger developed by the ventilator manufacturer for research purposes. The software downloaded all ventilator parameters and settings including peak inspiratory pressure, positive endexpiratory pressure, inspiratory and expiratory tidal volume, inspiratory and expiratory time, and fraction of inspired oxygen at a $0.5 \mathrm{~Hz}$ sampling rate. Data were retrieved with millisecond time stamps and exported as text files. The ventilator parameters correspond to the last inflation that occurred before the time stamp. Minute ventilation is calculated by the ventilator directly from the flow signal and is reported as a rolling mean over $30 \mathrm{~s}$ and includes both ventilator inflations and spontaneous breaths between inflations, if present. The software did not download the number of spontaneous breaths between SIMV inflations; however, their contribution to the total minute ventilation was reported as percentage. Clinical data were collected from electronic health care records. Blood gases were obtained via capillary sampling using heel pricks in all cases, as babies did not have arterial catheters. Gases were temperature corrected. Hypocapnia was defined as $\mathrm{pCO}_{2}$ below $35 \mathrm{kPa}(4.65 \mathrm{mmHg})$ [10]. 


\section{Data analysis}

Data were analyzed using Python (version 3.7.4, https://www. python.org) and its add-on packages. Anaconda (Continuum Analytics, http://docs.continuum.io/anaconda/pkg-docs) was installed on a MacBook Pro 2019 version, $2.4 \mathrm{GHz}$ quad-core i5 processor and $8 \mathrm{~Gb}$ RAM memory. Programming was done using Jupyter Notebook (version 7.10.2, http://ipython.org/ notebook.html). Data were represented, manipulated and analyzed using the NumPy (version 1.17.4, http://www.numpy. org) and pandas (version 0.25.3, http://pandas.pydata.org). Statistical analysis was performed using SciPy (version 1.3.1, www.scipy.org). For ventilator parameters showing normal distribution, arithmetic mean and standard deviation (SD) was calculated in each recording. For ventilator parameters with nonparametric distribution, median and interquartile range (IQR) was calculated in each recording. As these aggregate (mean or median) values themselves were not normally distributed within the two groups, the two groups were compared using nonparametric Mann-Whitney $U$ tests. Visualization was done using matplotlib (version 3.1.1, http://matplotlib.org). All software is open source and freely available. The Jupyter notebooks containing and explaining all steps of data processing and statistical analysis can be viewed on GitHub code repository at https://github.com/belteki/transport_HIE.

\section{Results}

We analyzed ventilator parameters and blood gases of infants ventilated using SIMV ventilation with VG $(n=28)$ or without VG $(n=8)$. The two groups were not significantly different for gestation, postnatal age, birth weight and basic clinical characteristics including Apgar score, respiratory severity score [22], or sedation (Table 1). Some infants received passive cooling by reducing the transport incubator's temperature; their proportion and temperatures were not different between the groups.

The median duration of ventilator recordings was 81 and $55 \mathrm{~min}$ in the SIMV-VG and SIMV group, respectively, with a total duration of $46.8 \mathrm{~h}$. As we collected the ventilator data with $0.5 \mathrm{~Hz}$ (1 data point every $2 \mathrm{~s}$ ) sampling rate, our analysis is based on $\sim 84,000$ data points. Descriptive statistics about the various ventilator parameters in the individual recordings are shown in Supplementary Tables 1 and 2. Comparison of the parameters between the two groups is shown in Table 2.

The expiratory tidal volumes of the ventilator inflations (VTemand) were significantly ( $p=0.01)$ lower in the group receiving SIMV-VG (median: $4.9 \mathrm{~mL} / \mathrm{kg}$, IQR: $4.6-5.3 \mathrm{~mL} /$ $\mathrm{kg}$ ) than in the group receiving SIMV without VG (median: $7.1 \mathrm{~mL} / \mathrm{kg}$, IQR: $5.3-8.0 \mathrm{~mL} / \mathrm{kg}$ ).
Table 1 Demographic and clinical details of the infants receiving SIMV-VG or SIMV ventilation.

\begin{tabular}{|c|c|c|c|}
\hline $\begin{array}{l}\text { Ventilation mode } \\
\text { Number of cases }\end{array}$ & $\begin{array}{l}\text { SIMV-VG } \\
\text { Count }\end{array}$ & $\begin{array}{l}\text { SIMV } \\
\text { Count }\end{array}$ & \\
\hline & 28 & 8 & \\
\hline \multirow{2}{*}{$\begin{array}{l}\text { Duration of recordings } \\
\text { (minutes) }\end{array}$} & Median (IQR) & Median (IQR) & $p^{\mathrm{a}}$ \\
\hline & $81(57-101)$ & $55(37-74)$ & 0.12 \\
\hline Demographic details & Mean (SD) & Mean (SD) & $p^{\mathrm{b}}$ \\
\hline Gestational age (weeks) & $38.9(1.4)$ & $38.9(1.3)$ & 0.92 \\
\hline Birth weight (grams) & $3463(584)$ & $3342(474)$ & 0.60 \\
\hline Postnatal age (hours) & $2.8(2.4)$ & $2.2(1.7)$ & 0.53 \\
\hline Apgar score & Median (IQR) & Median (IQR) & $p^{\mathrm{a}}$ \\
\hline At $5 \mathrm{~min}$ & $4(3-4)$ & $5(3-5)$ & \\
\hline At $10 \mathrm{~min}$ & $7(4-7)$ & $7(6-8)$ & \\
\hline \multicolumn{4}{|l|}{ Respiratory illness } \\
\hline $\begin{array}{l}\text { Respiratory severity } \\
\text { score (RSS) }\end{array}$ & $1.7(1.1-2.1)$ & $1.7(1.5-3.3)$ & 0.05 \\
\hline \multicolumn{4}{|l|}{$\begin{array}{l}\text { Capillary blood gas at the } \\
\text { beginning of transfer }\end{array}$} \\
\hline $\mathrm{pH}$ & $7.08(6.98-7.20)$ & $7.07(7.02-7.13)$ & 0.37 \\
\hline $\mathrm{pCO}_{2}(\mathrm{mmHg})$ & $48.6(39.6-61.2)$ & $48.9(36.2-59.5)$ & 0.29 \\
\hline $\begin{array}{l}\text { Actual base excess } \\
(\mathrm{mmol} / \mathrm{L})\end{array}$ & $\begin{array}{l}-15.0(-21.5 \\
\text { to }-11.1)\end{array}$ & $\begin{array}{l}-16.0(-18.6 \\
\text { to }-11.0)\end{array}$ & 0.46 \\
\hline $\begin{array}{l}\text { Therapeutic hypothermia } \\
\text { during transport }\end{array}$ & Count & Count & $p^{\mathrm{c}}$ \\
\hline Active cooling & 22 & 6 & 0.99 \\
\hline Passive cooling $^{\mathrm{d}}$ & 6 & 2 & \\
\hline Rectal temperature & Mean (SD) & Mean (SD) & $p^{\mathrm{b}}$ \\
\hline At the start of transport & $34.8(1.6)$ & $34.8(1.6)$ & 0.41 \\
\hline At the end of transport & $34.1(0.7)$ & $33.9(0.9)$ & 0.39 \\
\hline Sedative medication ${ }^{\mathrm{f}}$ & Count $(\%)$ & Count $(\%)$ & $p^{\mathrm{c}}$ \\
\hline None & $8(29 \%)$ & $2(25 \%)$ & 0.99 \\
\hline $\begin{array}{l}\text { Opioid (fentanyl/ } \\
\text { morphine) }\end{array}$ & $20(71 \%)$ & $5(63 \%)$ & \\
\hline $\begin{array}{l}\text { Benzodiazepine } \\
\text { (midazolam) }\end{array}$ & $1(4 \%)$ & $2(25 \%)$ & \\
\hline \multicolumn{4}{|l|}{ Anticonvulsant } \\
\hline None & $19(68 \%)$ & $7(88 \%)$ & 0.4 \\
\hline Phenobarbital & $9(32 \%)$ & $1(13 \%)$ & \\
\hline \multicolumn{4}{|l|}{ Muscle relaxants } \\
\hline None & $25(89 \%)$ & $5(63 \%)$ & 0.17 \\
\hline Atracurium/rocuronium & $3(11 \%)$ & $3(38 \%)$ & \\
\hline
\end{tabular}

For parametric variables arithmetic mean and standard deviations (SD) are shown. For nonparametric variables, median, and interquartile range (IQR) are given.

${ }^{a}$ Mann-Whitney $U$ test.

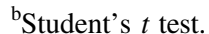

'Fisher's exact test.

${ }^{\mathrm{d}}$ Passive cooling included reducing or turning off the heat source of the incubator or the resuscitation equipment with monitoring of deep rectal temperature.

${ }^{\mathrm{e}}$ The respiratory severity score is calculated as $\mathrm{FiO}_{2} \times \mathrm{MAP}$ [22].

${ }^{\mathrm{f}}$ Some of the infants received more than one sedative medication. 
Table 2 Comparison of ventilators parameters in infants with hypoxicischemic encephalopathy during SIMV ventilation with or without volume guarantee.

\begin{tabular}{lccl}
\hline Parameter & $\begin{array}{l}\text { SIMV-VG } \\
\text { Group } \\
\text { median (IQR) }\end{array}$ & $\begin{array}{l}\text { SIMV } \\
\text { Group } \\
\text { median (IQR) }\end{array}$ & $\begin{array}{l}p \\
\text { value }^{\mathrm{a}}\end{array}$ \\
\hline VTemand (mL/kg) & $4.9(4.6-5.3)$ & $7.1(5.3-8.0)$ & 0.01 \\
VTset $(\mathrm{mL} / \mathrm{kg})$ & $4.4(3.9-4.8)$ & $\mathrm{n} / \mathrm{a}$ & \\
$\mathrm{PIP}\left(\mathrm{cmH}_{2} \mathrm{O}\right)$ & $10.7(7.8-17.2)$ & $17.5(16.6-19.4)$ & 0.02 \\
$\mathrm{MVspon} \%)$ & $42(14-59)$ & $32(18-42)$ & 0.29 \\
$\mathrm{FiO}_{2}(\%)$ & $21(21-26)$ & $21(21-46)$ & 0.29 \\
$\mathrm{MAP}\left(\mathrm{cmH} \mathrm{H}_{2} \mathrm{O}\right)$ & $6.2(5.1-7.7)$ & $7.0(6.7-8.1)$ & 0.08 \\
$\mathrm{MV}(\mathrm{L} / \mathrm{kg} / \mathrm{min})$ & $0.25(0.21-0.29)$ & $0.23(0.19-0.28)$ & 0.38 \\
$\mathrm{PEEP}\left(\mathrm{cmH} \mathrm{H}_{2} \mathrm{O}\right)$ & $5.1(5.0-6.0)$ & $5.8(5.0-6.0)$ & 0.20 \\
$\mathrm{RR}(1 / \mathrm{min})$ & $25.3(18.7-34.6)$ & $23.2(16.9-27.3)$ & 0.25 \\
\hline
\end{tabular}

For each patient, the mean (VTemand, VTset, PIP, PEEP, MAP, RR, $\mathrm{MV})$ or median $\left(\mathrm{FiO}_{2}, \mathrm{MVspon}\right)$ of ventilator parameter was calculated in the individual recordings (see Supplementary Tables 1 and 2). Data shown in this table are group medians and interquartile ranges (IQRs) of these aggregate (mean or median) values.

$\mathrm{FiO}_{2}$ fraction of inspired oxygen, $M A P$ mean airway pressure, $\mathrm{MV}$ minute volume, MVspon contribution of spontaneous breaths to total minute volume in percentage, $P E E P$ positive end-expiratory pressure, $P I P$ peak inflating pressure, $R R$ ventilator rate, SIMV synchronized intermittent mandatory ventilation, $S I M V-V G$ synchronized intermittent mandatory ventilation with volume guarantee, VTemand expiratory tidal volume of ventilator inflations, VTset set tidal volume target, n/a not applicable.

${ }^{a}$ Mann-Whitney $U$ test. $p$ values $<0.05$ were considered as significant, after correcting for multiple comparisons using the BenjaminiHochberg method.

Babies receiving SIMV-VG had significantly $(p=0.02)$ lower PIP with a larger variation (median: $10.7 \mathrm{cmH}_{2} \mathrm{O}$, IQR: $7.8-17.2 \mathrm{cmH}_{2} \mathrm{O}$ ) than those ventilated without $\mathrm{VG}$ (median: $17.5 \mathrm{cmH}_{2} \mathrm{O}$, IQR: $16.6-19.4 \mathrm{cmH}_{2} \mathrm{O}$ ).

Spontaneous breathing between the SIMV inflations contributed considerably to the total minute ventilation in both groups: its contribution to the total minute ventilation during SIMV-VG was 39\% (IQR: 18-52\%) and 30\% (IQR: $20-38 \%)$ during SIMV without VG $(p=0.29)$. The target tidal volume, ventilator rate, minute ventilation, $\mathrm{FiO}_{2}$, positive end-expiratory pressure, and mean airway pressure (MAP) were not different between the two groups (Table 2). In both groups, the majority of infants were ventilated with $\mathrm{FiO}_{2}<40 \%$ and MAP $<10 \mathrm{cmH}_{2} \mathrm{O}$ (Supplementary Fig. 1).

There was no significant difference between the two groups in capillary $\mathrm{pCO}_{2}$ at the end of the transport: the median [IQR] $\mathrm{pCO}_{2}$ was 46 [26-55] $\mathrm{mmHg}$ and 49 [27-59] $\mathrm{mmHg}$ for the SIMV-VG and SIMV groups $(p=0.42)$, respectively. Only $5(18 \%)$ infants ventilated with SIMVVG and $2(25 \%)$ infants ventilated with SIMV had an arrival $\mathrm{pCO}_{2}<35 \mathrm{mmHg}(4.65 \mathrm{kPa})$. An arrival $\mathrm{pCO}_{2}<30$ $\mathrm{mmHg}(4 \mathrm{kPa})$ was only seen in three infants, two in the
SIMV-VG group and one in the SIMV group. The change in $\mathrm{pCO}_{2}$ during transport was also not significantly different between the groups: the median [IQR] difference was 0.5 [ -9.0 to 8.0$] \mathrm{mmHg}$ for SIMV-VG recordings and -6.4 [ -12.3 to 4.6$] \mathrm{mmHg}$ for the SIMV recordings $(p=0.21)$. Babies with severe hypo- or hypercapnia at the beginning of transport tended to have larger changes in their $\mathrm{pCO}_{2}$ (Supplementary Fig. 2).

We further analyzed the SIMV-VG recordings to explore how close the actual expired tidal volumes were to the target values. For every inflation we determined VTdiff, the difference between the actual expiratory tidal volume and the target tidal volume and calculated the mean VTdiff for each recording. The group median $[\mathrm{IQR}]$ of the mean VTdiff was 0.33 [ -0.12 to 1.27$] \mathrm{mL} / \mathrm{kg}$. The mean VTdiff was $>1 \mathrm{~mL} / \mathrm{kg}$ in $9(32 \%)$ recordings. We also calculated Pdiff, the difference between the maximum allowed inflating pressure (Pmax) and the peak inflating pressure (PIP). The median Pdiff of the recordings ranged between -1.9 and $27.9 \mathrm{cmH}_{2} \mathrm{O}$. There were only $2(7 \%)$ recordings when the Pmax was within $5 \mathrm{cmH}_{2} \mathrm{O}$ of the PIP.

Among the babies ventilated with SIMV-VG there was a moderate but statistically significant inverse linear correlation between the contribution of the spontaneous breaths to the total minute ventilation and the mean PIP of the volume targeted ventilator inflations (Fig. 1). Babies who had $>50 \%$ of the total MV from spontaneous breathing $(n=9)$ had lower average PIP (group median: $7.7 \mathrm{cmH}_{2} \mathrm{O}$, IQR: $\left.7.1-8.1 \mathrm{cmH}_{2} \mathrm{O}\right)$ than babies $(n=19)$ who had $<50 \%$ of the MV from spontaneous breaths (group median: $12.2 \mathrm{cmH}_{2} \mathrm{O}$, IQR: $\left.10.0-18.9 \mathrm{cmH}_{2} \mathrm{O}, p<0.01\right)$. In babies with $\mathrm{PIP}<10$ $\mathrm{cmH}_{2} \mathrm{O} \quad(n=13)$, the VTemand and VTdiff were significantly $(p<0.01)$ larger than in babies with PIP $>10$ $\mathrm{cmH}_{2} \mathrm{O}(n=15)$ (Fig. 2a, b). Interestingly, $11(85 \%)$ of these infants received sedation during or shortly before the transfer and $2(15 \%)$ had also received muscle relaxant before the transport. These babies did not have adverse clinical events during transport and their $\mathrm{pCO}_{2}$ and $\mathrm{pH}$ immediately after transport was not different than that of the babies with higher $\left(>10 \mathrm{cmH}_{2} \mathrm{O}\right)$ mean PIP (Fig. 2c, d).

\section{Discussion}

In this retrospective study based on prospective computational data collection we analyzed ventilator parameters and blood gases during VG ventilation in infants undergoing therapeutic hypothermia for HIE; we compared babies receiving SIMV-VG with babies ventilated with SIMV only. To our knowledge this is the first report of VG ventilation in babies with HIE. Dassios et al. [23] studied ventilator parameters in babies with HIE and therapeutic hypothermia; however, the authors did not discuss if the 


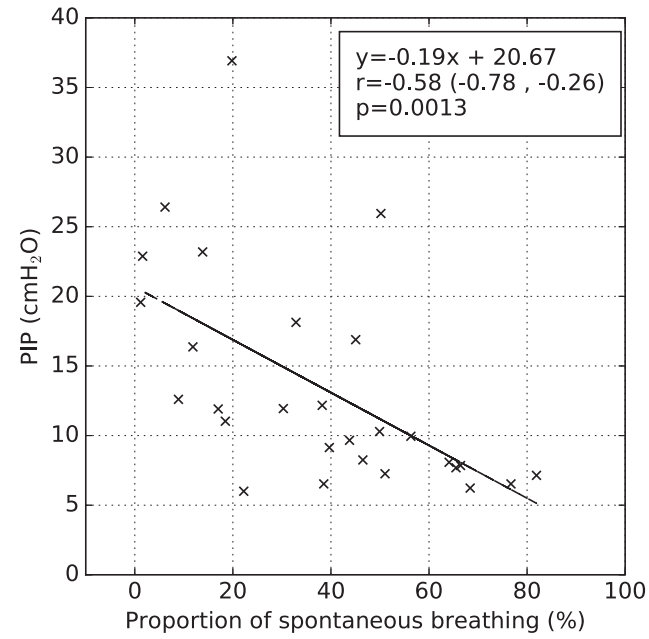

Fig. 1 Pearson's correlation between the infants' contribution to the total minute ventilation (MV) and the mean peak inflating pressure (PIP) during the transport in infants receiving SIMV-VG ventilation. Most babies whose spontaneous breathing accounted for over $50 \%$ of the total minute ventilation had very low $\left(<10 \mathrm{cmH}_{2} \mathrm{O}\right)$ PIP. Regression line, correlation coefficient $(r)$ and its $95 \%$ confidence interval is also shown. infants received volume targeted or pressure limited ventilation and their analysis was based on eight observations recorded manually over a 72-h period. We collected data computationally with $0.5 \mathrm{~Hz}$ sampling rate; therefore, our analysis is based on a dataset with thousands of data points.

VG ventilation of babies with HIE is potentially difficult for two reasons. First, these babies frequently hyperventilate to compensate for metabolic acidosis caused by the asphyxia and may develop hypocapnia. During VG ventilation the ventilator stops an inflation if the inspiratory tidal volume significantly exceeds the target VT; however, babies can continue breathing from the continuous flow in the ventilator circuit [19]. Despite this, we found the babies ventilated with VG had lower tidal volumes and maintained their average tidal volume close to the target value.

Despite the lower tidal volumes, minute ventilation and $\mathrm{pCO}_{2}$ at the end of transfer was not different between the groups. There are several possible explanations for this. First, during SIMV babies can breathe spontaneously between the ventilator inflations, particularly if the ventilator rate is set low. The tidal volumes of these spontaneous
Fig. 2 Comparison of ventilator parameters and blood gases in babies receiving SIMV-VG ventilation during transport with mean $\mathrm{PIP}<\mathbf{1 0}$ $\mathrm{cmH}_{2} \mathrm{O}$ and $>10 \mathrm{cmH}_{2} \mathrm{O}$. a The expired tidal volume of ventilator inflations (VTemand). b The difference between the actual and targeted expired tidal volume (VTdiff). c pCO $\mathrm{pC}_{2}$ and d $\mathrm{pH}$ immediately after the transport. Babies with low PIP had significantly higher VTemand and VTdiff and did not develop hypercapnia or acidosis during the transport. Horizontal lines represent medians, boxes represent interquartile ranges, whiskers show full range of values. Mean values are shown by black diamonds.
A

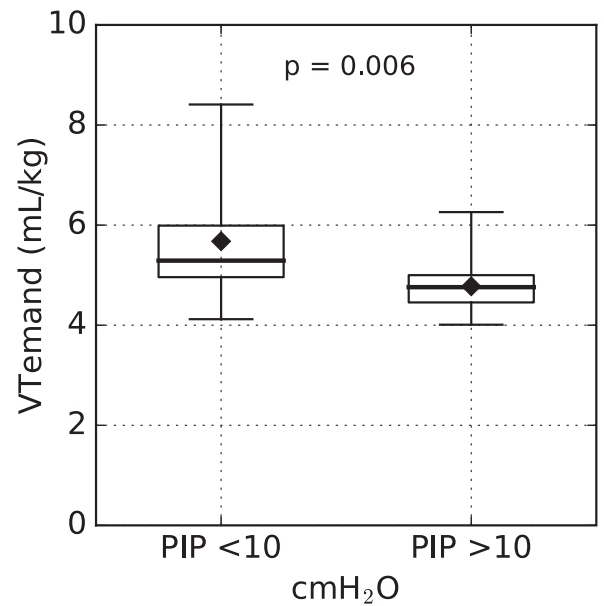

C

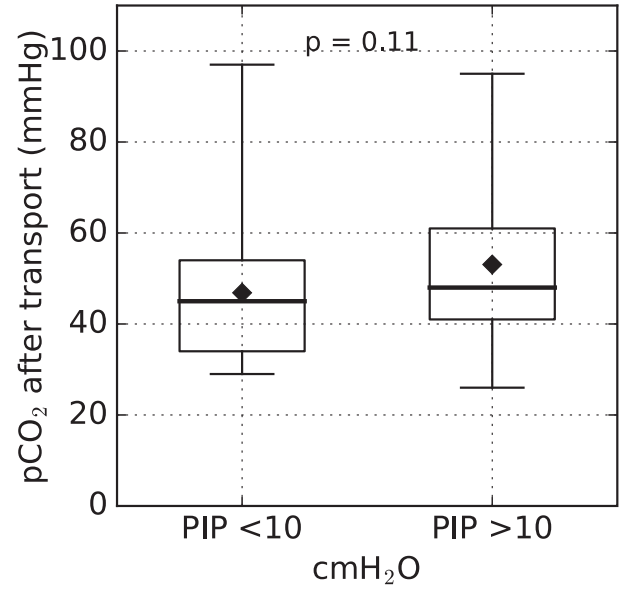

B

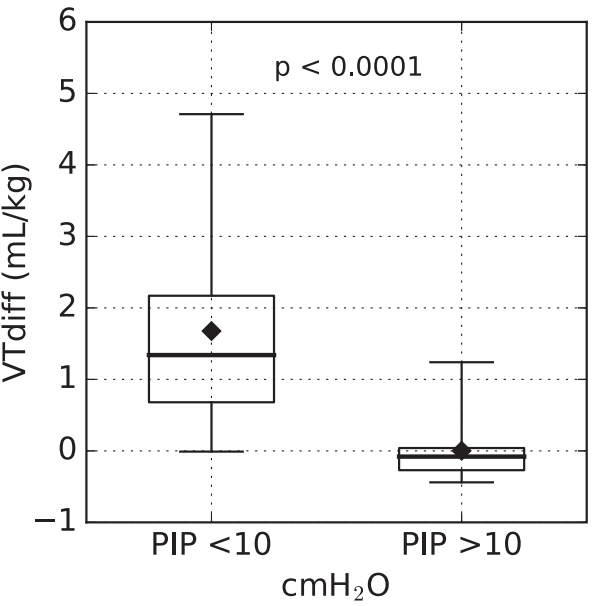

D

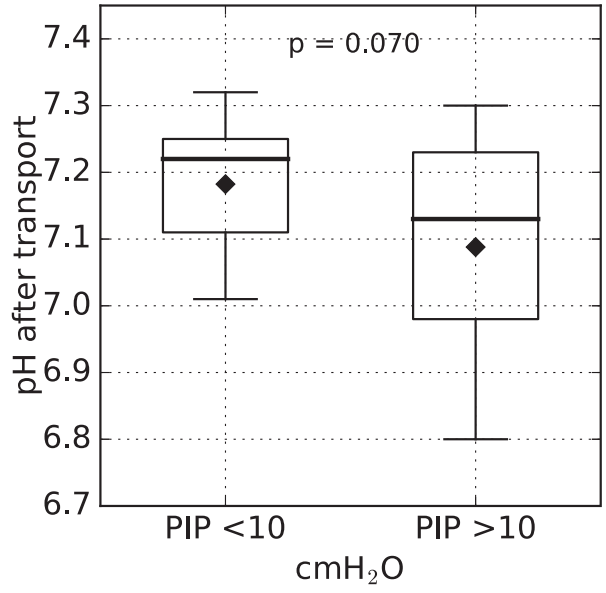


breaths are controlled solely by the infant even when VG is used. In our dataset the SIMV rate was $<30 /$ min for most babies. We found that in some babies, spontaneous breaths contributed more than $50 \%$ to the total minute ventilation even when sedation was used. Therefore, the lower tidal volumes of ventilator inflations during SIMV-VG may have had a limited effect. However, using a higher SIMV rate or using SIPPV ventilation mode (which delivers a ventilator inflation with each attempted breath) would have been likely to result in a higher minute ventilation and lower $\mathrm{pCO}_{2}$ irrespective whether VG is used or not. Second, it has been reported during SIMV-VG infants breathe more between ventilator inflations than during SIMV with the same rate but without VG [24]. Third, the study was short, limited to the transport period. Finally, our study may have been underpowered to detect the potential benefit of VG on $\mathrm{pCO}_{2}$ at the end of transport. Based on data from a previous study [8] from the same transport service, and assuming the variability of $\mathrm{pCO}_{2}$ seen in our data, even if there was a $50 \%$ reduction in hypocapnia after of transport, it would have required a balanced sampled size of 120 patients.

The second concern with VG ventilation in term infants with HIE and strong breathing effort is that the VG algorithm lowers the PIP to try and maintain the VTemand near the set level and hence the ventilator's driving pressure (PIP-PEEP) and contribution to the work of breathing in these babies, is frequently minimal [19]. Concerns have been raised in review articles that this leads to exhaustion, hypercapnia, and metabolic acidosis [20], although we could not find any report presenting data to support this hypothesis. We also point out that while this situation is superficially similar to endotracheal continuous positive airway pressure, during VG the ventilator compares the actual expired tidal volume to the target at every inflation and if the set VT is not delivered with minimal PIP due to exhaustion or other causes, the PIP is increased immediately. In our dataset we found that PIPs below $10 \mathrm{cmH}_{2} \mathrm{O}$ (driving pressure $<4-5 \mathrm{cmH}_{2} \mathrm{O}$ ) occurred mostly in babies whose spontaneous breathing contributed to their ventilation by more than $50 \%$. These babies frequently exceeded the set VT due to hyperventilation and did not develop hypercapnia or acidosis. However, based on these data we cannot rule out these adverse events during longer ventilation periods or in babies with different clinical characteristics (e.g., in preterm infants).

The main strength of our study is the prospective computational data collection. Moreover, our cohort was uniform as all recordings happened on the first day of life during ex utero transfer to a cooling center.

A limitation of our study is that it was a retrospective analysis, the sample size was relatively low, and it was limited to the duration of interhospital transfer lasting no more than $1-2 \mathrm{~h}$. Also, we had only two capillary gases for each transfer (one at the beginning and one the end) rather than serial arterial blood gases. Capillary $\mathrm{pCO}_{2}$ values may have been significantly higher than arterial $\mathrm{pCO}_{2}$ values in babies who had peripheral vasoconstriction due to therapeutic hypothermia. Although there was no difference between the groups in the number of babies receiving sedation, the actual level of sedation and spontaneous breathing effort could have been different. Finally, no clinical or long-term outcomes have been studied. The use of VG ventilation in these babies needs to be established in future clinical studies.

In summary, we found the VG ventilation in babies with HIE and therapeutic hypothermia reduces the tidal volume of ventilator inflations but does not influence minute ventilation or $\mathrm{CO}_{2}$ elimination. Using VG in babies with $\mathrm{HIE}$ leads to low PIP, particularly in babies with strong breathing effort; however, the low PIPs did not result in worsening of the acidosis in these infants.

Acknowledgements We thank to Roland Hotz, Rainer Kühner, and the engineers of Acutronic for their help to export data from the Fabian ventilator. We thank to Dr Amanda Ogilvy-Stuart for her advice and comments on the paper.

Author contributions GB designed the study, wrote the computer programs to analyze ventilator data, performed the data analysis, and drafted the paper. $\mathrm{LL}, \mathrm{AB}$, and $\mathrm{ZS}$ performed the neonatal transfers and collected the clinical data. CJM helped with interpreting the findings. All authors revised and approved the final paper.

\section{Compliance with ethical standards}

Conflict of interest The authors declare that they have no conflict of interest.

Publisher's note Springer Nature remains neutral with regard to jurisdictional claims in published maps and institutional affiliations.

\section{References}

1. Douglas-Escobar M, Weiss MD. Hypoxic-ischemic encephalopathy: a review for the clinician. JAMA Pediatr. 2015;169:397-403.

2. Liu L, Oza S, Hogan D, Chu Y, Perin J, Zhu J, et al. Global, regional, and national causes of under-5 mortality in 2000-15: an updated systematic analysis with implications for the Sustainable Development Goals. Lancet. 2016;388:3027-35.

3. Kurinczuk JJ, White-Koning M, Badawi N. Epidemiology of neonatal encephalopathy and hypoxic-ischaemic encephalopathy. Early Hum Dev. 2010;86:329-38.

4. Jacobs SE, Berg M, Hunt R, Tarnow-Mordi WO, Inder TE, Davis PG. Cooling for newborns with hypoxic-ischemic encephalopathy. Cochrane Database Syst Rev. 2013;1:CD003311.

5. Natarajan G, Pappas A, Shankaran S. Outcomes in childhood following therapeutic hypothermia for neonatal hypoxic-ischemic encephalopathy (HIE). Semin Perinatol. 2016;40:549-55.

6. Szakmar E, Jermendy A, El-Dib M. Respiratory management during therapeutic hypothermia for hypoxic-ischemic encephalopathy. J Perinatol. 2019;39:763-73. 
7. Pfister RH, Bingham P, Edwards EM, Horbar JD, Kenny MJ, Inder $\mathrm{T}$, et al. The Vermont Oxford Neonatal Encephalopathy Registry: rationale, methods, and initial results. BMC Pediatr. 2012;12:84.

8. Szakmar E, Kovacs K, Meder U, Bokodi G, Szell A, Somogyvari $\mathrm{Z}$, et al. Asphyxiated neonates who received active therapeutic hypothermia during transport had higher rates of hypocapnia than controls. Acta Paediatr. 2018;107:1902-8.

9. Nadeem M, Murray D, Boylan G, Dempsey EM, Ryan CA. Blood carbon dioxide levels and adverse outcome in neonatal hypoxicischemic encephalopathy. Am J Perinatol. 2010;27:361-5.

10. Pappas A, Shankaran S, Laptook AR, Langer JC, Bara R, Ehrenkranz RA, et al. Eunice Kennedy Shriver National Institute of Child Health and Human Development Neonatal Research Network. Hypocarbia and adverse outcome in neonatal hypoxicischemic encephalopathy. J Pediatr. 2011;158:752-8.

11. Lopez Laporte MA, Wang H, Sanon PN, Barbosa Vargas S, Maluorni J, Rampakakis E, et al. Association between hypocapnia and ventilation during the first days of life and brain injury in asphyxiated newborns treated with hypothermia. J Matern Fetal Neonatal Med. 2019;32:1312-20.

12. Klinger G, Beyene J, Shah P, Perlman M. Do hyperoxaemia and hypocapnia add to the risk of brain injury after intrapartum asphyxia? Arch Dis Child Fetal Neonatal Ed. 2005;90:49-52.

13. Greisen G, Munck H, Lou H. Severe hypocarbia in preterm infants and neurodevelopmental deficit. Acta Paediatr Scand. 1987; 76:401-4.

14. Fritz KI, Zubrow A, Mishra OP, Delivoria-Papadopoulos M. Hypercapnia-induced modifications of neuronal function in the cerebral cortex of newborn piglets. Pediatr Res. 2005;57:299-304.

15. Gupta A, Keszler M. Survey of ventilation practices in the neonatal intensive care units of the United States and Canada: use of volume targeted ventilation and barriers to its use. Am J Perinatol. 2019;36:484-9.

16. Klingenberg C, Wheeler KI, McCallion N, Morley CJ, Davis PG. Volume-targeted versus pressure-limited ventilation in neonates. Cochrane Database Syst Rev. 2017;10:CD003666.

17. Peng W, Zhu H, Shi H, Liu E. Volume-targeted ventilation is more suitable than pressure-limited ventilation for preterm infants: a systematic review and meta-analysis. Arch Dis Child Fetal Neonatal Ed. 2014;99:F158-65.

18. Bhat P, Chowdhury O, Shetty S, Hannam S, Rafferty GF, Peacock $\mathrm{J}$, et al. Volume-targeted versus pressure-limited ventilation in infants born at or near term. Eur J Pediatr. 2016;175:89-95.

19. Keszler M, Abubakar K. Volume guarantee: stability of tidal volume and incidence of hypocarbia. Pediatr Pulmonol. 2004;38:240-5.

20. Keszler M. Volume-targeted ventilation: one size does not fit all. Evidence-based recommendations for successful use. Arch Dis Child Fetal Neonatal Ed. 2019;104:F108-12.

21. Azzopardi DV, Strohm B, Edwards AD, Dyet L, Halliday HL, Juszczak E, et al. Moderate hypothermia to treat perinatal asphyxial encephalopathy. N Engl J Med. 2009;361:1349-58.

22. Iyer NP, Mhanna MJ. Non-invasively derived respiratory severity score and oxygenation index in ventilated newborn infants. Pediatr Pulmonol. 2013;48:364-9.

23. Dassios T, Austin T. Respiratory function parameters in ventilated newborn infants undergoing whole body hypothermia. Acta Paediatr. 2014;103:157-61.

24. Herrera CM, Herrera CM, Gerhardt T, Claure N, Everett R, Musante $\mathrm{G}$, et al. Effects of volume-guaranteed synchronized intermittent mandatory ventilation in preterm infants recovering from respiratory failure. Pediatrics. 2002;110: $529-33$. 